\title{
Stability and Trajectories Analysis of a Fractional Generalization of Simple Pendulum Dynamic Equation
}

\author{
Ibrahima N'Doye and Taous-Meriem Laleg Kirati
}

\begin{abstract}
In this paper, we present the dynamics of the simple pendulum by using the fractional-order derivatives. Equations of motion are proposed for cases without input and external forcing. We use the fractional-order Euler-Lagrange equations to obtain the fractional-order dynamic equation of the simple pendulum. We perform equilibria analysis, indicate the conditions where stability dynamics can be observed for both integer and fractional-order models. Finally, phase diagrams have been plotted to visualize the effect of the fractional-order derivatives.
\end{abstract}

Index Terms-Simple pendulum model, nonlinear systems, fractional-order dynamics, fractional-order Euler-Lagrangian, stability analysis, trajectories analysis, numerical simulations.

\section{INTRODUCTION}

Fractional calculus is a generalization of classical differentiation and integration to arbitrary (non-integer) order. In recent years fractional calculus has gained significant attention as one of the topics that can be applied to a variety of engineering fields [1]. Many studies and researches in controls have concentrated on using fractional calculus for modeling systems or designing controllers showing that the fractional-order differential equations are, at least, as stable as their integer-order counterparts [2].

Fractional calculus has gained a lot of importance and potential applications in several areas of science and engineering, such as fluid mechanics, viscoelasticity, biology, physics and engineering.

Fractional dynamics and fractional variational principles has been used as an efficient tool in modeling complex physical systems. The advantage of fractional calculus over classical integer-order calculus is its non-local and memory properties. It is well known that the integer-order differential operator is a local operator but the fractional-order differential operator is non-local. This means that the next state of a system depends not only upon its current state but also upon all of its historical states. This more realistic interpretation explains why fractional calculus has become more and more popular. Recently, the existence, uniqueness and dissipativity for a class of nonlinear dynamical systems including systems with fractional damping have been investigated [3]. A numerical integration method has been applied to a nonlinear pendulum with fractional damping as well as to a nonlinear pendulum suspended on an extensible string [3]. The formulation of

Ibrahima N'Doye and Taous-Meriem Laleg-Kirati are with Computer, Electrical and Mathematical Sciences and Engineering Division (CEMSE), King Abdullah University of Science and Technology (KAUST), Thuwal 23955-6900, Saudi Arabia. (email: ibrahima.ndoye@kaust.edu.sa; taousmeriem.laleg@kaust.edu.sa) the fractional-order Euler-Lagrange problem has recently drawn the attention of many authors in their works [4], [5]. The fractional variational principles and the fractional EulerLagrange were obtained in [6], the fractional constrained Lagrangian and Hamiltonian were analyzed in [7], [8]. The classical fields with fractional-order derivatives were investigated by using the fractional Lagrangian formulation and the fractional Euler-Lagrange equations were obtained in [9].

Fractional Lagrangian of the two-electric pendulum has been studied in [10] and its numerical analysis has been carried out. Recently the fractional Euler-Lagrange equations for the fractional Bateman-Feshbach-Tikochinsky oscillator, which is a non-conservative dissipative system has been studied in [11].

Another aspect which requires a passing mention is how evolutionary and swarm algorithms and other computational intelligence paradigms can be improved with the help of fractional calculus. All of the swarm algorithms and their variants use position and velocity update equations. If a fractional version of velocity can be used instead of the standard integer-order versions, there is a scope for arriving at insightful results and improved algorithms. This is because there is an inherent long-range memory in fractional-order differentiation [12]. This kind of velocity update can take care of previous positions of the particle and influence the present velocity based on the past history. Thus, instead of only relying on the past best position of the particle, the fractional differentiation operator can offer a weighted version of the past history of the position of the particle in swarm based search and optimization algorithms [12]. The long-term memory is an important characteristic of fractional differentiation and is also a prominent difference between fractional differentiation and integer-order differentiation.

In this paper, we study fractional-order simple pendulum model. The motion of material objects is not always in two or three dimensions; it rather depends on restraint conditions in relativistic mechanics. Researchers have extended integer space into fractional space as the dimension of space plays an important role in areas such as quantum field theory and Casimir effect. Even more interestingly, the dimension of the real world was measured experimentally and found to be $3 \pm 10^{-6}$. This is in accordance with general relativity, which states that gravitational fields are curvatures in space-time, instead of being entities in a flat space-time [13], [14]. With the above motivation and based on the nonlinear ordinary differential equations of the simple pendulum, we study 
and analyze a fractional-order model which describes the temporal dynamics of the angular position and the angular velocity.

This paper is organized as follows: In section II, the basic definition and preliminaries of fractional calculus are briefly introduced. In section III, we present the integer-order simple pendulum model. Section IV is about the concept of fractional-order simple pendulum model, stability analysis, and simulation examples. The phase plane analysis with various initial conditions and fractional-order derivatives is presented in order to analyze the stability of the angular position and the angular velocity. The discussion of our numerical simulations is provided in section V. Concluding remarks are given in section VI.

\section{PRELiminary}

\section{A. Definition of fractional derivatives}

In this paper, we use the Grünwald-Letnikov derivative [2]. The latter is defined by the fractional differentiation-order $\alpha$ of function $f(t)$ with respect to $t$ and the terminal value 0 :

${ }_{0} D_{t}^{\alpha} f(t)=\frac{\mathrm{d}^{\alpha} f(t)}{\mathrm{d} t^{\alpha}}=\lim _{t \rightarrow 0} h^{-\alpha} \sum_{j=0}^{\left[\frac{t}{n}\right]}(-1)^{j}\left(\begin{array}{l}\alpha \\ j\end{array}\right) f(t-j h)$,

with $n \in \mathbb{N}$ and $\alpha \in \mathbb{R}^{+}$, [.] means the integer part.

In the rest of the paper, we use an operator ${ }_{0} D_{t}^{\alpha}$ to represent the Grünwald-Letnikov derivative with zero initial value history.

\section{B. Numerical solution of fractional-order differential equa- tions}

For numerical simulation of the fractional-order systems, the Grünwald-Letnikov method [15], [16] based on the Adams-Bashforth-Moulton type predictor-corrector scheme [17] has been proposed and widely used.

The formula for the explicit numerical approximation of the $\alpha^{t h}$ derivative at the points $k h$ (see also [15], [16], [18]) is given by

$$
\left(k-L_{m} / h\right) D_{k h}^{\alpha} f(t) \approx h^{-\alpha} \sum_{j=0}^{k}(-1)^{j}\left(\begin{array}{l}
\alpha \\
j
\end{array}\right) f\left(t_{k}-j\right),
$$

where $L_{m}$ is the memory length, $t_{k}=k h, h$ is the time step of the calculation and $(-1)^{j}\left(\begin{array}{l}\alpha \\ j\end{array}\right)$ are binomial coefficients $c_{j}^{(\alpha)}(j=0,1, \cdots)$ which can be computed as

$$
c_{0}^{(\alpha)}=1, \quad c_{j}^{(\alpha)}=\left(1-\frac{1+\alpha}{j}\right) c_{j-1}^{(\alpha)} .
$$

Numerical solution of the fractional-order differential equation

$$
D_{t}^{\alpha} y(t)=f(y(t), t)
$$

can be expressed as [16]

$$
y\left(t_{k}\right)=f\left(y\left(t_{k}\right), t_{k}\right) h^{\alpha}-\sum_{j=1}^{k} c_{j}^{(\alpha)} f\left(t_{k}-j\right) .
$$

Equation (5) is nonlinear with respect to finding $y\left(t_{k}\right)$ and can be solved using any suitable method for such equations.

\section{Fractional-order systems}

The fractional-order linear time-invariant system can be represented by the following state-space model

$$
\left\{\begin{array}{l}
{ }_{0} D_{t}^{\alpha} x(t)=A x(t)+B u(t) \\
y(t)=C x(t)
\end{array}\right.
$$

where $x(t) \in \mathbb{R}^{n}$ is the state vector, $u(t) \in \mathbb{R}^{m}$ is the control input vector and $y(t) \in \mathbb{R}^{p}$ is the measured output. $A \in \mathbb{R}^{n \times m}, B \in \mathbb{R}^{n \times n}, C \in \mathbb{R}^{p \times n}$ are constant real matrices and $\alpha=\left[\alpha_{1}, \alpha_{2}, \cdots, \alpha_{n}\right]^{T}$ are the fractional-orders. If $\alpha_{1}=\alpha_{2}=\cdots=\alpha_{n}$ system (6) is called a commensurate order system; otherwise it is an non-commensurate order system.

Generally, we consider the following non-commensurate fractional-order nonlinear system in the form [16]

$$
\left\{\begin{array}{l}
{ }_{0} D_{t}^{\alpha_{i}} x_{i}(t)=f_{i}\left(x_{1}(t), x_{2}(t), \cdots, x_{n}(t), t\right) \\
x_{i}(t)=c_{i}, \quad i=1,2, \cdots, n
\end{array}\right.
$$

where $c_{i}$ are initial conditions, or in its vector representation

$$
\mathbf{D}^{\alpha} \mathbf{x}=\mathbf{f}(\mathbf{x}),
$$

where $\alpha=\left[\alpha_{1}, \alpha_{2}, \cdots, \alpha_{n}\right]^{T}$ for $0<\alpha_{i}<2, \quad(i=$ $1,2, \cdots, n)$ and $\mathbf{x} \in \mathbb{R}^{n}$. The equilibrium points of system (6) are calculated by solving the following equation

$$
\mathbf{f}(\mathbf{x})=0
$$

and we suppose that $x^{*}=\left(x_{1}^{*}, x_{2}^{*}, \cdots, x_{n}^{*}\right)$ is an equilibrium point of system (6).

\section{Stability of fractional-order systems}

It has been shown that linear fractional-order system (6) is stable if the following condition is satisfied [19], [20] for $0<\alpha \leqslant 1$, and for $1 \leqslant \alpha<2$ [21] (also if the triplet $A, B, C$ is minimal)

$$
|\arg (\operatorname{eig}(A))|>\alpha \frac{\pi}{2}
$$

where eig $(A)$ represents the eigenvalues of matrix $A$.

In the case of nonlinear fractional-order systems, the equilibrium points are asymptotically stable for $\alpha_{1}=\alpha_{2}=\cdots=$ $\alpha_{n} \equiv \alpha$ if all the eigenvalues $\lambda_{i}(i=1,2, \cdots, n)$ of the Jacobian matrix $\mathbf{J}=\partial \mathbf{f} / \partial \mathbf{x}$, where $f=\left[f_{1}, f_{2}, \cdots, f_{n}\right]^{T}$ evaluated at the equilibrium, satisfy the condition [22], [23]

$$
|\arg (\operatorname{eig}(\mathbf{J}))|=\left|\arg \left(\lambda_{i}\right)\right|>\alpha \frac{\pi}{2}, \quad i=1,2 \cdots, n \text {. }
$$

The problem of fractional non-commensurate order systems can be solved in different ways according to the literature (e.g. [19], [24], [25], [26]). When we consider the noncommensurate fractional-order system $\alpha_{1} \neq \alpha_{2} \neq \cdots \neq \alpha_{n}$ and suppose that $m$ is the least common multiple (LCM) of the denominators $u_{i}^{\prime} s$ of $\alpha_{i}^{\prime} s$, where $\alpha_{i}=v_{i} / u_{i}, v_{i}, u_{i} \in \mathbb{Z}^{+}$ for $(i=1,2, \cdots, n)$ and we set $\gamma=1 / m$, system (8) is asymptotically stable if

$$
|\arg (\lambda)|>\gamma \frac{\pi}{2}
$$

for all roots $\lambda$ of the following equation

$$
\operatorname{det}\left(\operatorname{diag}\left(\left[\begin{array}{ll}
\lambda^{m \alpha_{1}} & \lambda^{m \alpha_{2}} \cdots \lambda^{m \alpha_{n}}
\end{array}\right]\right)-\mathbf{J}\right)=0 .
$$




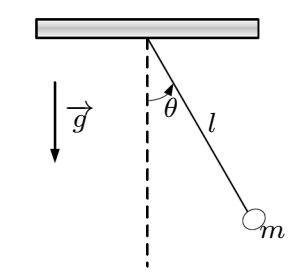

Fig. 1. Simple pendulum.

A necessary stability condition for the fractional-order systems (8) to remain chaotic is keeping at least one eigenvalue $\lambda$ in the unstable region [22]. The number of saddle points and eigenvalues for one-scroll, double-scroll and multi-scroll attractors was exactly described in [23]. Suppose that the unstable eigenvalues of scroll saddle points are: $\lambda=\rho \pm j \beta$. The necessary condition to exhibit one scroll attractor of system (8) is for the eigenvalues $\lambda$ to remain in the unstable region [23]. The condition for commensurate derivative orders is

$$
\alpha>\frac{2}{\pi} \tan ^{-1}\left(\frac{|\beta|}{\rho}\right) \text {. }
$$

Otherwise, one of the equilibrium points becomes asymptotically stable and attracts the nearby trajectories. According to (13), this necessary condition is mathematically equivalent to

$$
\frac{\pi}{2 m}-\min (|\arg (\lambda)|) \geqslant 0
$$

\section{ClASSICAL MODEL OF SIMPLE PENDULUM}

The simple pendulum consists of a massless string with one end attached to a weight and the other end fixed. When an initial push is given, the pendulum will swing back and forth under the influence of gravity. A schematic of the simple pendulum system is shown in Fig. 1. The analysis of the simple pendulum is important in numerous areas of maths and physics. It is an important example because its equation of motion is nonlinear; perhaps it is one of the most studied and analyzed nonlinear systems. The equation of the free undamped simple pendulum motion is given by the following known state space representation

$$
\left\{\begin{array}{l}
\dot{\theta}(t)=\omega(t) \\
\dot{\omega}(t)=-\frac{g}{l} \sin \theta(t)
\end{array}\right.
$$

where $\theta(t)$ is the angular position, $\omega(t)$ the angular velocity, $g$ the acceleration due to gravity and $l$ is the length of the pendulum.

Equation (16), although straightforward in appearance, is in fact rather difficult to solve because of the nonlinearity of the term $\sin \theta(t)$.

The kinetic energy of the pendulum is given by

$$
T=\frac{1}{2} m \ell^{2} \dot{\theta}^{2}
$$

and choosing the zero potential energy point when $\theta=0$ (see Fig. 1), the gravitational potential energy is

$$
U=m g \ell(1-\cos \theta) \text {. }
$$

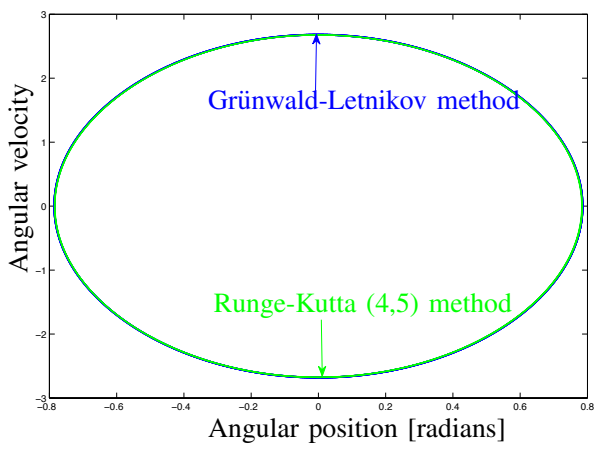

Fig. 2. Comparison of two numerical methods: Grünwald-Letnikov method and Runge-Kutta $(4,5)$ method.

As a result, the entire Lagrangian function is

$$
L=\frac{1}{2} m \ell^{2} \dot{\theta}^{2}-m g \ell(1-\cos \theta),
$$

and the Euler-Langrange equation for $\theta$ is

$$
\frac{\partial L}{\partial \theta}+\frac{d}{\mathrm{~d} t}\left(\frac{\partial L}{\partial \dot{\theta}}\right)=0
$$

which is equivalent to

$$
\ddot{\theta}+\frac{g}{\ell} \sin \theta=0 .
$$

Let $x=\left[\begin{array}{ll}x_{1} & x_{2}\end{array}\right]^{T}=\left[\begin{array}{ll}\theta & \omega\end{array}\right]^{T}$, then we can rewrite system (16) into the following form

$$
\left\{\begin{array}{l}
\dot{x}_{1}(t)=x_{2}(t) \\
\dot{x}_{2}(t)=-\frac{g}{l} \sin x_{1}(t) .
\end{array}\right.
$$

System (22) is equivalent to system (16). The equilibrium points are given by

$$
x_{2}=0, \quad \sin x_{1}=0
$$

which leads to the equilibrium points $E_{1}=(0[2 \pi], 0)$ and $E_{2}=(\pi[2 \pi], 0)$. Physically, these points correspond to the pendulum resting exactly at the vertical up and down positions. The Jacobian matrix of system (22) is

$$
\mathbf{J}=\left[\begin{array}{cc}
0 & 1 \\
-\frac{g}{l} \cos x_{1}^{*} & 0
\end{array}\right]
$$

for the equilibrium $E^{*}=\left(x_{1}^{*}, x_{2}^{*}\right)$.

System (22) has two fixed points (equilibria) $E_{1}=(0,0)$ and $E_{2}=(\pi, 0)$ and their stability can be studied by computing the corresponding eigenvalues. For the constant parameters $g=9.81 \mathrm{~m} / \mathrm{s}^{2}$ and $l=0.8 \mathrm{~m}$, the equilibrium $E_{1}$ has the eigenvalues $\lambda_{1,2}= \pm 3.5018 j$ and $E_{2}$ has the eigenvalues $\lambda_{1,2}= \pm 3.5018$. Hence, the equilibrium point $E_{1}$ is a center point and the equilibrium $E_{2}$ is a saddle point.

In the following section, we focus on theoretically and numerically studying the stability analysis of the fractionalorder simple pendulum.

\section{FRACTIONAL-ORDER MODEL OF SIMPLE PENDULUM}

The fractional-order form of the Lagrangian equation (19) has the following form

$$
L^{F}=\frac{1}{2} m \ell^{2}\left[D^{\alpha} \theta\right]^{2}-m g \ell(1-\cos \theta) .
$$


Now, to obtain the Euler-Lagrange equation for the generalized coordinate $\theta$ we use

or equivalently as

$$
\frac{\partial L^{F}}{\partial \theta}+D^{\alpha} \frac{\partial L^{F}}{\partial D^{\alpha} \theta}=0
$$

$$
D^{\alpha}\left[D^{\alpha} \theta\right]+\frac{g}{\ell} \sin \theta=0
$$

As $\alpha \rightarrow 1$, we obtain the classical Euler-Lagrange equation (21).

Now consider the fractional-order simple pendulum system described by the following fractional-order differential equations

$$
\left\{\begin{array}{l}
D^{\alpha_{1}} x_{1}(t)=x_{2}(t), \\
D^{\alpha_{2}} x_{2}(t)=-\frac{g}{l} \sin x_{1}(t),
\end{array}\right.
$$

where $x_{1}$ reflects the temporal dynamics of the angular position, $x_{2}$ mimics the temporal dynamics of the angular velocity, and $\alpha_{1}$ and $\alpha_{2}$ are the derivatives orders. The total order of the system is $\bar{\alpha}=\left(\alpha_{1}, \alpha_{2}\right)$.

Numerical solution of the fractional-order simple pendulum system (27) is given as follows

$$
\left\{\begin{array}{l}
x_{1}\left(t_{k}\right)=\left(x_{1}\left(t_{k-1}\right)\right) h^{\alpha_{1}}-\sum_{j=1}^{k} c_{j}^{\left(\alpha_{1}\right)} x_{1}\left(t_{k}-j\right), \\
x_{2}\left(t_{k}\right)=\left(-\frac{g}{l} \sin \left(x_{1}\left(t_{k-1}\right)\right)\right) h^{\alpha_{2}}-\sum_{j=1}^{k} c_{j}^{\left(\alpha_{2}\right)} x_{2}\left(t_{k}-j\right),
\end{array}\right.
$$

for which $T_{s}$ is the simulation time by setting $N=\left[T_{s} / h\right]$, $k=1,2, \cdots, N$, and $\left(x_{1}(0), x_{2}(0), x_{3}(0)\right)$ are the initial conditions. The binomial coefficients $c_{j}^{\left(\alpha_{i}\right)}, \forall i$ are calculated according to relation (3).

The phase plane analysis is a graphical method for studying dynamic systems. The basic idea of this method is to generate motion trajectories corresponding to various initial conditions, and then to examine the qualitative features of the trajectories. In such a way, information concerning stability of the system can be obtained. In this section, we use the tools of phase plane analysis to acquire insights on the effects of fractional-order derivative with nonlinearities and compare the motion trajectories of simple pendulum system between fractional-order derivative and their integer-order counterpart with different initial conditions and various fractional-order derivatives.

For illustration, we can compare the proposed numerical method, called the Grünwald-Letnikov method, with the explicit Runge-Kutta method known as ode45 routine in Matlab. As can be seen in Fig. 2, the proposed numerical algorithm has approximately the same order of accuracy as the Runge-Kutta algorithm for time step 0.0005 with constant parameters $g=9.81 \mathrm{~m} / \mathrm{s}^{2}, l=0.8 \mathrm{~m}$ and derivative orders $\alpha_{1}=\alpha_{2}=1$.

\section{A. Commensurate order}

Let us consider the commensurate order system (27) with $\alpha_{1}=\alpha_{2}=\widetilde{\alpha}=0.92$ and the following system parameters $g=9.81 \mathrm{~m} / \mathrm{s}^{2}$ and $l=0.8 \mathrm{~m}$. We should investigate the stability of equilibrium point $E_{1}$.
The stability of the equilibrium point $E_{1}$ is of importance. If it were stable, non zero states might be attracted towards it. The second fixed point $E_{2}$ is not hyperbolic, so no conclusions can be drawn from the linear analysis. For the constant parameters $g=9.81 \mathrm{~m} / \mathrm{s}^{2}$ and $l=0.8 \mathrm{~m}$, the equilibrium points are the same as in the case of integerorder system. The stability can be investigated according to condition (11).

Case $\alpha_{1}=\alpha_{2}=\widetilde{\alpha}=0.92$ : According to the stability condition (11), where $\widetilde{\alpha}=0.92$, we have eigenvalue

$$
\alpha \frac{\pi}{2}<\left|\arg \left(\lambda_{1,2}\right)\right|=1.5708,
$$

for the equilibrium $E_{1}$.

The characteristic equation of the linearized system (27) evaluated at the equilibrium point $E_{1}$ is

$$
\begin{gathered}
\lambda^{184}+\frac{981}{80}=0, \quad \text { with } \\
\frac{\pi}{2 m}<\min _{i}\left(\left|\arg \left(\lambda_{i}\right)\right|\right)=0.0171,
\end{gathered}
$$

where $m=100$ (LCM of orders denominator), then we can confirm the asymptotical stability of system (27). The numerical simulations support this result.

Case $\alpha_{1}=\alpha_{2}=\bar{\alpha}=0.98$ : The characteristic equation of the linearized system (27) evaluated at the equilibrium point $E_{1}$ where $\bar{\alpha}=0.98$ is

$$
\begin{gathered}
\lambda^{196}+\frac{981}{80}=0, \quad \text { with } \\
\frac{\pi}{2 m}<\min _{i}\left(\left|\arg \left(\lambda_{i}\right)\right|\right)=0.0160,
\end{gathered}
$$

where $m=100$ (LCM of orders denominator), then we can confirm the asymptotical stability of system (27). The numerical simulations support this result.

In Figs. 3, 4 and 5 are shown the phase plane diagram for system (27) with initial conditions $\left(x_{1}(0), x_{2}(0)\right)=(\pi / 4,0)$, $\left(x_{1}(0), x_{2}(0)\right)=(\pi / 2,0)$ and $\left(x_{1}(0), x_{2}(0)\right)=(\pi / 12,6)$ respectively. This shows that when $\alpha_{1}=\alpha_{2}=\alpha=1$ the system oscillates, and when $\alpha_{1}=\alpha_{2}=\bar{\alpha}=0.98$ and $\alpha_{1}=\alpha_{2}=\widetilde{\alpha}=0.92$ the system converges asymptotically to the equilibrium point.

Case $\alpha_{1}=\alpha_{2}=\bar{\alpha}=0.9$ : The characteristic equation of the linearized system (27) evaluated at the equilibrium point $E_{1}$ where $\bar{\alpha}=0.9$ is

$$
\begin{gathered}
\lambda^{18}+\frac{981}{80}=0, \quad \text { with } \\
\frac{\pi}{2 m}<\min _{i}\left(\left|\arg \left(\lambda_{i}\right)\right|\right)=0.1745,
\end{gathered}
$$

where $m=10$ (LCM of orders denominator), then we can confirm the asymptotical stability of system (27). The numerical simulations support this result.

Case $\alpha_{1}=\alpha_{2}=\widetilde{\alpha}=0.8$ : The characteristic equation of the linearized system (27) evaluated at the equilibrium point $E_{1}$ where $\widetilde{\alpha}=0.8$ is

$$
\begin{gathered}
\lambda^{16}+\frac{981}{80}=0, \quad \text { with } \\
\frac{\pi}{2 m}<\min _{i}\left(\left|\arg \left(\lambda_{i}\right)\right|\right)=0.1964,
\end{gathered}
$$

where $m=10$ (LCM of orders denominator), then we can confirm the asymptotical stability of system (27). The 
numerical simulations support this result.

In Figs. 6, 7, 8 and 9 are shown the phase plane diagrams for system (27) with initial conditions $\left(x_{1}(0), x_{2}(0)\right)=$ $(\pi / 4,0), \quad\left(x_{1}(0), x_{2}(0)\right)=(\pi / 2,0), \quad\left(x_{1}(0), x_{2}(0)\right)=$ $(\pi / 12,6)$ and $\left(x_{1}(0), x_{2}(0)\right)=(\pi / 6,1)$ respectively. This shows that when $\alpha_{1}=\alpha_{2}=\alpha=1$ the system oscillates, and when $\alpha_{1}=\alpha_{2}=\bar{\alpha}=0.9$ and $\alpha_{1}=\alpha_{2}=\widetilde{\alpha}=0.8$ the system converges asymptotically to the equilibrium point.

\section{B. Non-commensurate order}

When we assume the different orders of derivatives in the fractional-order pendulum system (27), i.e. $\alpha_{1} \neq \alpha_{2}$, we get a general non-commensurate order system. We should investigate the stability of equilibrium point $E_{1}$.

The stability of the equilibrium point $E_{1}$ is of importance. If it were stable, non zero states might be attracted towards it. The second fixed point $E_{2}$ is not hyperbolic, so no conclusions can be drawn from the linear analysis. For the constant parameters $g=9.81 \mathrm{~m} / \mathrm{s}^{2}$ and $l=0.8 \mathrm{~m}$, the equilibrium points are the same as in the case of integerorder system. The stability can be investigated according to condition (13).

The characteristic equation of the linearized system (27) evaluated at the equilibrium point $E_{1}$ where $\alpha_{1}=0.95$ and $\alpha_{2}=0.85$ is

$$
\begin{gathered}
\lambda^{180}+\frac{981}{80}=0, \quad \text { with } \\
\frac{\pi}{2 m}<\min _{i}\left(\left|\arg \left(\lambda_{i}\right)\right|\right)=0.0175,
\end{gathered}
$$

where $m=100$ (LCM of orders denominator), then we can confirm the asymptotical stability of system (27). The numerical simulations support this result.

In Figs. 10 and 11 are shown the phase plane diagrams for system (27) with initial conditions $\left(x_{1}(0), x_{2}(0)\right)=(\pi / 4,0)$ and $\left(x_{1}(0), x_{2}(0)\right)=(\pi / 2,0)$ respectively. This shows that when $\alpha_{1}=\alpha_{2}=\alpha=1$ the system oscillates, and when $\alpha_{1}=0.95$ and $\alpha_{2}=0.85$ the system converges asymptotically to the equilibrium point.

\section{Discussions}

The simulation results for the phase plane fractional-order simple pendulum with constant parameters $g=9.81 \mathrm{~m} / \mathrm{s}^{2}$, $l=0.8 \mathrm{~m}$ and the commensurate and non-commensurate fractional-order derivatives are shown in Figs. $3-11$ with various initial conditions. Under the assumption of null air resistance or the absence of other forces and starting with some arbitrary initial conditions, the pendulum oscillates and progressively stops at the vertical with the fractionalorder derivative, and continues to swing indefinitely with the integer-order derivative case.

The points $(0[2 \pi], 0)$ are stable equilibrium points. These points become attractors for long times in the fractional-order derivative case and the phase paths will spiral towards them. Figs. $3-11$ show that the fractional-order simple pendulum system converges asymptotically to the equilibrium point. This comes from the fact that the total mechanical energy of the fractional-order pendulum system is progressively dissipated, so that the pendulum comes to rest at a position of minimal energy, until eventually the system settle down to the equilibrium point. The local asymptotical stability implies the convergence of mechanical energy to zero. We have seen that the presence of a fractional differential-order in a differential equation can lead to a notable increase in the complexity of the observed behavior, and the solution continuously depends on all the previous states. We observed that the fractional-order model without damping term describes vanishing responses to zero when time tends to infinity while the friction-free integer model generates oscillating responses. This analysis might also bring an insight to neglect the friction terms in non-integer models. For accuracy tests, the energy is numerically determined and compared with the accumulated energy dissipation. The energy of a mechanical system with fractional-order derivatives like in the case of a viscoelastic medium [27] is not uniquely defined by the equations of motion. The energy decreases with respect to its original value in the fractional-order case. In contrast with the classical integer-order dissipation model, the energy does not decay monotonically. It is however possible to redefine the energy by adding a history-dependent part in such a way that the new energy decreases monotonically or is conserved.

In view of the numerical simulation applied we can seen that the energy of the simple pendulum with the integerorder derivative case does not decay monotonically due to the implicit inertial and elastic effects. In the case of fractional-order derivative, it is however possible to construct a monotonically decaying energy. Such an energy is a history functional, or, equivalently, it depends on internal states variables which cannot be determined by the numerical algorithm adopted in this paper.

By choosing a simple pendulum system with two degrees of freedom for numerical analysis we are able to demonstrate the interaction of fractional-order derivative with nonlinear interactions. This paper also serves as a demonstration of a method for integrating systems of ordinary differential equations and fractional-order derivatives. This method uses a recently developed Grünwald-Letnikov method [15] based on the Adams-Bashforth-Moulton type predictor-corrector scheme [17].

\section{CONCLUSION}

In this paper, a fractional-order simple pendulum has been presented. It has been shown that the presence of a fractional differential-order in a differential equation can lead to a notable increase in the complexity of the observed behavior, and the solution continuously depends on all the previous states. We observed that the fractional-order model without damping term describes vanishing responses to zero when time tends to infinity while the friction-free integer-order model generates oscillating responses. This analysis might bring an insight to neglect the friction terms in non-integer models. The numerical simulations show that the angular position and velocity converge to their equilibrium points. However, experiments should be conducted to obtain more 


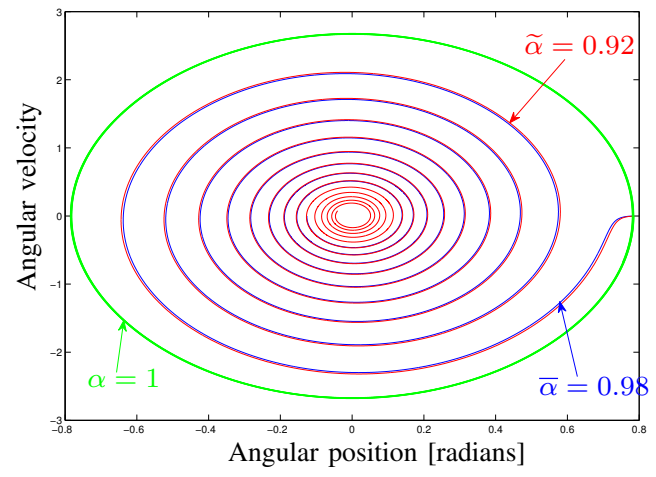

Fig. 3. Phase plane diagram for system (27) with initial conditions $\left(x_{1}(0), x_{2}(0)\right)=(\pi / 4,0)$. This shows that when $\alpha_{1}=\alpha_{2}=\alpha=1$ the system oscillates, and when $\alpha_{1}=\alpha_{2}=\bar{\alpha}=0.98$ and $\alpha_{1}=\alpha_{2}=\widetilde{\alpha}=0.92$ the system converges asymptotically to the equilibrium point.

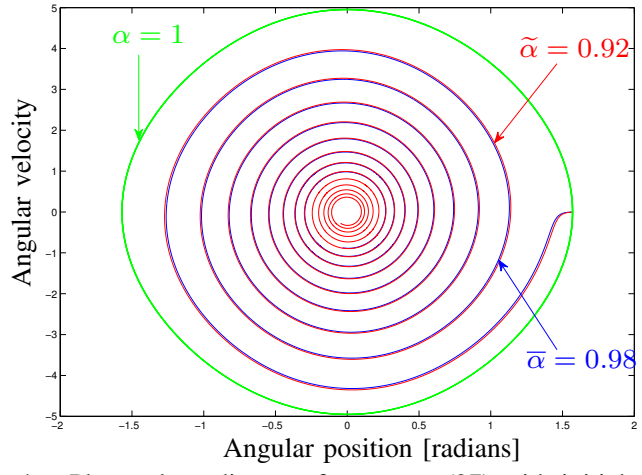

Fig. 4. Phase plane diagram for system (27) with initial conditions $\left(x_{1}(0), x_{2}(0)\right)=(\pi / 2,0)$. This shows that when $\alpha_{1}=\alpha_{2}=\alpha=1$ the system oscillates, and when $\alpha_{1}=\alpha_{2}=\bar{\alpha}=0.98$ and $\alpha_{1}=\alpha_{2}=\widetilde{\alpha}=0.92$ the system converges asymptotically to the equilibrium point.

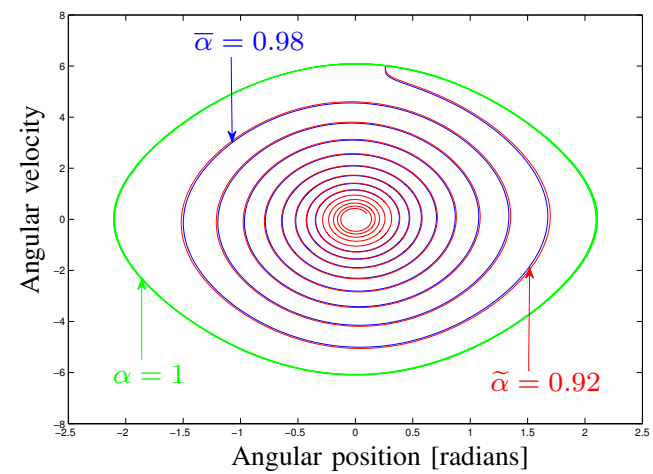

Fig. 5. Phase plane diagram for system (27) with initial conditions $\left(x_{1}(0), x_{2}(0)\right)=(\pi / 12,6)$. This shows that when $\alpha_{1}=\alpha_{2}=\alpha=1$ the system oscillates, and when $\alpha_{1}=\alpha_{2}=\bar{\alpha}=0.98$ and $\alpha_{1}=\alpha_{2}=\widetilde{\alpha}=0.92$ the system converge asymptotically to the equilibrium point.

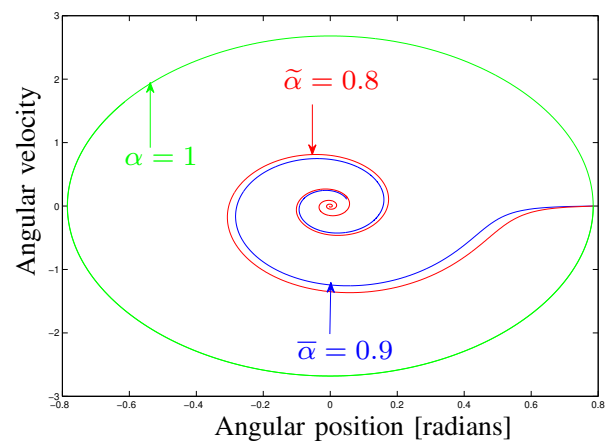

Fig. 6. Phase plane diagram for system (27) with initial conditions $\left(x_{1}(0), x_{2}(0)\right)=(\pi / 4,0)$. This shows that when $\alpha_{1}=\alpha_{2}=\alpha=1$ the system oscillates, and when $\alpha_{1}=\alpha_{2}=\widetilde{\alpha}=0.8$ and $\alpha_{1}=\alpha_{2}=$ $\bar{\alpha}=\alpha=0.9$ the system converges asymptotically to the equilibrium point.

realistic results about whether fractional-order models are more accurate than integer-order models. This analysis may be useful for better understanding and control of such system.

\section{ACKNOWLEDGEMENT}

The research reported herein is supported by the King Abdullah University of Science and Technology (KAUST).

\section{REFERENCES}

[1] R. Hilfer, Applications of Fractional Calculus in Physics. Singapore: World Scientific Publishing, 2001.

[2] I. Podlubny, Fractional Differential Equations. New York: Academic Press, 1999.

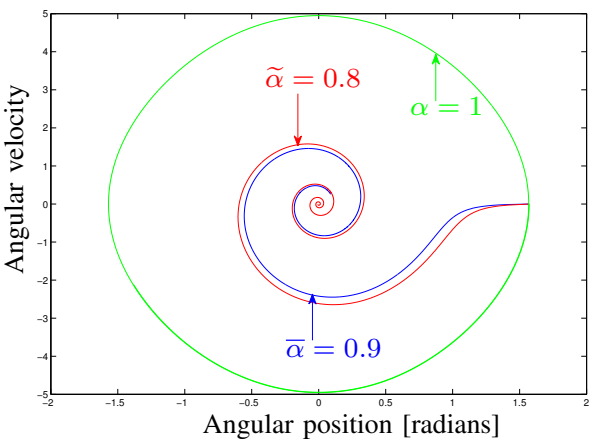

Fig. 7. Phase plane diagram for system (27) with initial conditions $\left(x_{1}(0), x_{2}(0)\right)=(\pi / 2,0)$. This shows that when $\alpha_{1}=\alpha_{2}=\alpha=1$ the system oscillates, and when $\alpha_{1}=\alpha_{2}=\widetilde{\alpha}=0.8$ and $\alpha_{1}=\alpha_{2}=$ $\bar{\alpha}=\alpha=0.9$ the system converges asymptotically to the equilibrium point.

[3] M. Seredynska and H. Hanyga, "Nonlinear differential equations with fractional damping with applications to the 1dof and 2dof pendulum," Acta Mathematica, vol. 176, pp. 169-183, 2005.

[4] D. Baleanu, K. Diethelm, E. Scalas, and J. J. Trujillo, Fractional Calculus Models and Numerical Methods. Series on Complexity, Nonlinearity and Chaos, World Scientific, 2012.

[5] D. Baleanu and J. J. Trujillo, "On exact solutions of a class of fractional Euler-Lagrange equations," Nonlinear Dynamics, vol. 52, pp. 331-335, 2007.

[6] P. O. Agrawal, "Formulation of Euler-Lagrange equations for fractional variational problems," Journal of Mathematical Analysis and Applications, vol. 272, pp. 368-379, 2002.

[7] S. Muslih and D. Baleanu, "Hamiltonian formulation of systems with linear velocities within Riemann-Liouville fractional derivatives," Journal of Mathematical Analysis and Applications, vol. 304, pp. 2632-2636, 2005. 


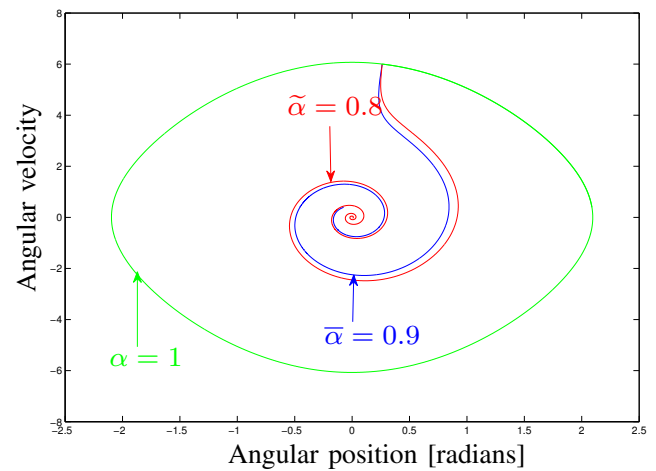

Fig. 8. Phase plane diagram for system (27) with initial conditions $\left(x_{1}(0), x_{2}(0)\right)=(\pi / 12,6)$. This shows that when $\alpha_{1}=\alpha_{2}=$ $\alpha=1$ the system oscillates, and when $\alpha_{1}=\alpha_{2}=\widetilde{\alpha}=0.8$ and $\alpha_{1}=\alpha_{2}=\bar{\alpha}=\alpha=0.9$ the system converges asymptotically to the equilibrium point.

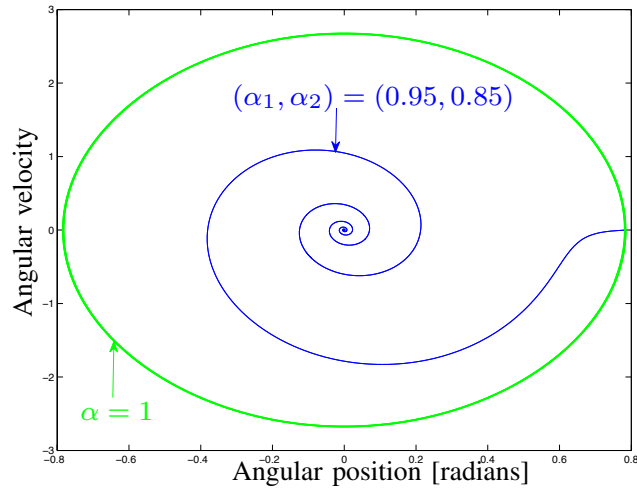

Fig. 10. Phase plane diagram for system (27) with initial conditions $\left(x_{1}(0), x_{2}(0)\right)=(\pi / 4,0)$. This shows that when $\alpha_{1}=\alpha_{2}=\alpha=1$ the system oscillates, and when $\alpha_{1}=0.95$ and $\alpha_{2}=0.85$ the system converges asymptotically to the equilibrium point.

[8] D. Baleanu and T. Avkar, "Lagrangians with linear velocities within Riemann-Liouville fractional derivatives," Nuovo Cimento della Societa Italiana di Fisica B, vol. 119, no. 1, pp. 73-79, 2004.

[9] D. Baleanu and I. S. Muslih, "Lagrangian formulation of classical fields within Riemann-Liouville fractional derivatives," Physica Scripta, vol. 72, no. 2, pp. 119-121, 2005.

[10] D. Baleanu, J. H. Asad, and I. Petras, "Fractional-order two-electric pendulum," Romanian reports in Physics, vol. 64, pp. 907-914, 2012.

[11] D. Baleanu, H. J. Asad, and I. Petráš, "Fractional Bateman-Feshbach Tikochinsky oscillator," Communications in Theoretical Physics, vol. 61, no. 2, pp. 221-225, 2014.

[12] I. Pan and S. Das, Intelligent Fractional Order Systems and Control - An Introduction. Berlin Heidelberg: Springer-Verlag, 2013.

[13] E. Anli and I. Ozkol, "Classical and fractional-order analysis of the free and forced double pendulum," Engineering, vol. 2, pp. 935-949, 2010.

[14] S. I. Muslih and D. Baleanu, "Fractional Euler-Lagrange equations of motion in fractional space," Journal of Vibration and Control, vol. 13, pp. 1209-1216, 2007.

[15] I. Petrás, "A note on the fractional-order Volta system," Commun Nonlinear Sci. Numer. Simulat., vol. 15, pp. 384-393, 2010.

[16] I. Petráš, Fractional-Order Nonlinear Systems : Modeling, Analysis and Simulation. Berlin: Springer, 2011.

[17] W. Deng, "Short memory principle and a predictor-corrector approach for fractional differential equations," J. Comput. Appl. Math., vol. 206, pp. 174-188, 2007.

[18] L. Dorckák, "Numerical models for simulation of the fractional-order control systems," Tech. Rep. UEF-04-94, Institute of Experimental Physics, Academy of Sciences, Slovakia, 1994.

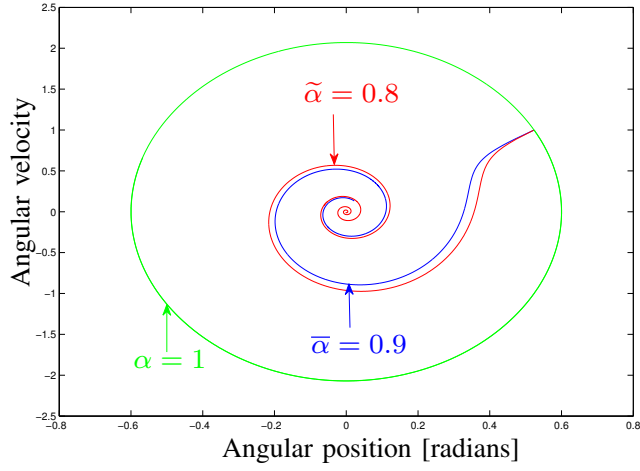

Fig. 9. Phase plane diagram for system (27) with initial conditions $\left(x_{1}(0), x_{2}(0)\right)=(\pi / 6,1)$. This shows that when $\alpha_{1}=\alpha_{2}=\alpha=1$ the system oscillates, and when $\alpha_{1}=\alpha_{2}=\widetilde{\alpha}=0.8$ and $\alpha_{1}=\alpha_{2}=$ $\bar{\alpha}=\alpha=0.9$ the system converges asymptotically to the equilibrium point.

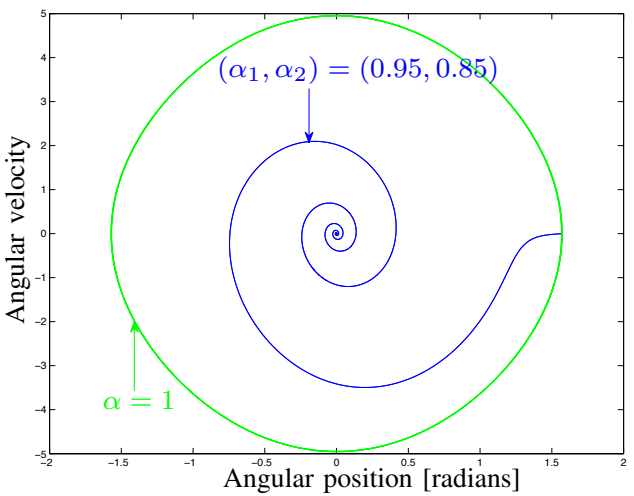

Fig. 11. Phase plane diagram for system (27) with initial conditions $\left(x_{1}(0), x_{2}(0)\right)=(\pi / 2,0)$. This shows that when $\alpha_{1}=\alpha_{2}=\alpha=1$ the system oscillates, and when $\alpha_{1}=0.95$ and $\alpha_{2}=0.85$ the system converges asymptotically to the equilibrium point.

[19] D. Matignon, "Stability results for fractional differential equations with applications to control processing," in Proc. IEEE-IMACS Syst. Man Cyber. Conf., (Lille, France), 1996.

[20] D. Matignon, "Generalized fractional differential and difference equations : stability properties and modelling issues," in Proc. Mathematical Theory of Networks and Systems Symposium, (Padova, Italy), 1998.

[21] J. Sabatier, M. Moze, and C. Farges, "On stability of fractional order systems," in Proc. IFAC Workshop on Fractional Differentiation and its Application, (Ankara, Turkey), 2008.

[22] S. M. Tavazoei and M. Haeri, "A necessary condition for double scroll attractor existence in fractional-order systems," Physics Letter A, vol. 367, pp. 102-113, 2007.

[23] S. M. Tavazoei and M. Haeri, "Limitations of frequency domain approximation for detecting chaos in fractional order systems," Nonlinear Analysis, vol. 69, pp. 1299-1320, 2008.

[24] Y. Li, Y. Chen, and I. Podlubny, "Mittag-Leffler stability of fractional order nonlinear dynamic systems," Automatica, vol. 45, pp. 19651969, 2009.

[25] Y. Li, Y. Chen, and I. Podlubny, "Stability of fractional-order nonlinear dynamic systems : Lyapunov direct method and generalized Mittag-Leffler stability," Computers \& Mathematics with Applications, vol. 59, pp. 1810-1821, 2010.

[26] A. Oustaloup, J. Sabatier, P. Lanusse, R. Malti, and P. M. et al. "An overview of the CRONE approach in system analysis, modeling and identification, observation and control," in World Congress IFAC, (Seoul, Korea), pp. 14254-14265, 2008.

[27] A. Morro and M. Vianello, "Minimal and maximal free energy for materials with memory," Boll. UMI, vol. 97, pp. 45-55, 1990. 